\title{
An efficient non-parametric analysis of recognition memory'
}

\author{
Ir win Pollack, UNIVERSTTY OF MICHIGAN Donald A. Norman, HARVARD UNIVESITY \\ Eugene Galanter, UNIVERSTY OF WASHINGTON
}

\begin{abstract}
Abstraet
A method for efficient evaluation of the results of recognition memory experiments is illustrated. The method is based upon the area under the iso-mnemonic curve relating correct and incorrect acceptances obtained by means of ratings.
\end{abstract}

\section{Problem}

In recognition studies, the criterion for acceptance of an item as an "old" item is in the hands of S, not E. This creates a methodological difficulty in obtaining a measure of recognition performance which is independent of S's criterion. The present note considers an efficient procedure for analysis of recognition which circumvents this difficulty.

\section{Method}

A non-parametric analysis of recognition experiments has been presented for the case in which only a single recognition score is available (Pollack \& Norman, 1964). That analysis approximates an analysis of recognition experiments with important theoretical properties, which requires several recognition scores (Green, 1964).

The experimental procedure for efficiently obtaining a number of recognition scores under a given experimental condition is the use of a rating scale. For example, Table 1 illustrates typical results when a 6point rating scale is employed, ranging from +3 ("positive this item is anold item") through -3 ("positive this item is a new item"). The second and fourth columns illustrate the division of ratings among 100 old items and 100 new items, respectively. Had Semployed a criterion for an o response between the +2 and +3 ratings, we assume the proportion of o responses would have been the proportion of +3 ratings. Had Semployed a criterion for an 0 response between the +1 and +2 ratings, we assume the proportion of o responses would have been the sum of the +2 and the +3 ratings, etc. Column 3 thus represents the proportion of correct acceptances (o responses to old items) and is obtained by successively cumulating the entries of column 2 . Column 5 represents the proportion of incorrect acceptances (o responses to new items) and is obtained by cumulating the entries of column 4. In this way, $(n-1)$ recognition scores are efficiently obtained with an $\mathrm{n}$-point rating scale for a single set of responses. (The final score is determined.)

A plot of the paired recognition scores of columns 3 and 5, an "iso-mnemonic curve," sweeps over different response criteria. Paralleling Green (1964), the area under the curve is selected as the measure of recognition performance. By the trapezoidal rule the area under the curve of the results of Table 1 is 0.898 .
Table 1. Example of Application of Ratings to Recognition of Old and New Items.

$\begin{array}{ccccc}\text { Rating } & \text { (R|old) } & \sum \text { (o|old) } & \text { (R|new) } & \sum \text { (o|new) } \\ +3 & 43 & .43 & 2 & .02 \\ +2 & 16 & .59 & 3 & .05 \\ +1 & 21 & .80 & 10 & .15 \\ -1 & 12 & .92 & 17 & .32 \\ -2 & 5 & .97 & 18 & .50 \\ -3 & 3 & 1.00 & 50 & 1.00\end{array}$

\section{Experiment I}

Paced by a metronome, $\mathrm{S}$ exposed 100 monosyllabic words typed upon index cards. The interval between successive words ranged from 1 to $8 \mathrm{sec}$. in separate experimental sessions. After presentation of each set of 100 words, $S$ was given a sheet of 200 words, consisting of 100 old words and 100 new words in a scrambled order. S employed a 6-point rating scale under self-paced conditions. The order of presentation of the intervals and of the word sets were scrambled among 6 Ss (university students) in separate experimental sessions. Following these tests, 5 Ss were tested with 3digit random numbers.

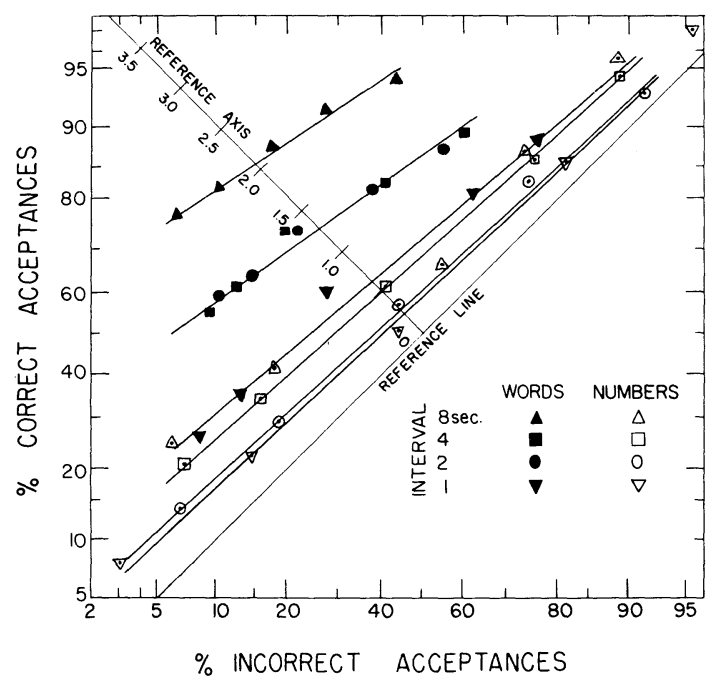

Fig. 1. Iso-mnemonic curves for the recognition of words and numbers under four intervals between successive items. The coordinates are scaled in terms of the cumulative normal distribution. The $45^{\circ}$ reference line represents a chance level of performance. The reference axis represents the locus of points in thich the sum of correct and incorrect acceptances adds to 1.0. It is scaled in standard score form. 
The results of Experiment I are presented in Fig. 1 as iso-mnemonic curves upon coordinates of the integrated normal distribution. Individual results preserve the general relationships of the pooled results. The results are also presented in Table 2 in terms of the area under the iso-mnemonic curve when plotted upon linear coordinates.

Recognition performance improves as the interval between successive items is increased and is consistently higher for words than for numerals under corresponding conditions. The slopes of the iso-mnemonic curves progressively decrease as the curves become displaced toward higher performance levels, i.e. toward the upper left corner of the graph.

\section{Experiment II}

CVC nonsense syllables typed on index cards were presented in sets of 20 under paced and unpaced conditions. In the unpaced conditions, $\mathrm{S}$ was given either 20,40 , or $80 \mathrm{sec}$. to examine the set of 20 items. These conditions are represented in Table 2 as U20, U40, and U80. Five paced conditions are differentiated in terms of the time per item, the number of presentations of the set of 20 items, and the total presentation period. Thus, 1-1-20 refers to a presentation schedule of 1 sec. per item, one presentation of the set of 20 items, and a total presentation period of $20 \mathrm{sec}$.

Table 2. Areas under Iso-mnemonic Curve.

$\begin{array}{cccc}\text { Exp. } & \text { Period } & \text { Words } & \text { Numerals } \\ \text { I } & 1 \text { sec. } & .70 & .55 \\ & 2 \text { sec. } & .80 & .58 \\ 4 \text { sec. } & .79 & .63 \\ & 8 \text { sec. } & .88 & .66 \\ & \text { Condition } & \text { S: AH } & \text { S: RH } \\ & \text { II } & .70 & .73 \\ & \text { U20** } & .73 & .59 \\ & 1-2-40 & .78 & .69 \\ & 2-1-40 & .84 & .78 \\ \text { U40 } & .80 & .73 \\ & 2-2-80 & .89 & .78 \\ 4-1-80 & .89 & .72 \\ \text { U80 } & .87 & .73\end{array}$

* Listed successively are: sec. per item; number of presentations of set of 20 items; and total presentation period in sec.

** Unpaced
Following presentation of the materials, S scored data of another study for $5 \mathrm{~min}$. A sheet of 40 items, 20 old and 20 new, served as the test. S employed a 5-point rating scale under self-paced conditions. Each experimental condition was run within a single experimental session. Six experimental sesisions, plus a practice session, were carried out with two Ss: A.H. was relatively experienced in psychophysical testing; R.H. was relatively unexperienced.

The results of Experiment II are presented in Table 2 in terms of the area under the iso-mnemonic curve. Results for A.H. suggest that recognition period, irrespective of the temporal distribution within the period. This result is consistent with that of Bugelski \& Rickwood (1963) for paired associate learning. Results for R.H. are more variable, but performance tends to improve with the total presentation period.

\section{Diseussion}

The area measure of recognition memory is nonparametric in that no specific assumption is made about the form of the iso-mnemonic curve. Nevertheless, because of the formal similarity between recognition memory and signal detection studies, it is instructive to examine the theory of signal detection (TSD) as a parametric alternative. Indeed, Egan (1958) examined recognition memory in these terms. TSD provides a simple measure of discriminability when results plot as lines of unit slope upon the representation of Fig. 1. When unit slope is not obtained, as in the present study and that of Egan, an arbitrary measure of discriminability must be selected, such as the intersection with the reference axis. Thus, no special advantage accrues by the use of the parametric alternative of TSD. References

BUGELSKI, B. R., \& RICKWOOD, J. Presentation time, total time, and mediation in paired-associate learning: self-pacing. J. exp. Psychol., 1963, 65, 616-617.

EGAN, J. P. Recognition memory and the operating characteristic. Indiana University, Hearing and Communication Laboratory, AFCRC TN 58 51, AD-152650. 15 June 1958.

GREEN, D. M. General prediction relating yes-no and forced-choice results. J. Acoust. Soc. Amer., 1964, 36, 1042 (A).

POLLACK, I., \& NORMAN, D. A. A non-parametric analysis of recognition experiments. Psychon. Sci., $1964,1,125-126$.

\section{Note}

1. The authors acknowledge the assistance of Marcia Feingold for programming the IBM 7090 computer for calculation of areas under the iso-mnemonic curves. 